This small series of tive cases. with the possible exception of No. 4167, shows that tuberculosis of the lungs may reveal signs at the beginning and end of periods of 15 to 25 years. Fach patient had pursued an indoor occupation, except No. 2648, who had probably been advised to take up the work of a 'bus conductor in order to get open air.

This lecture must be concluded with an expression of the hope that others will be found who will supply evidence of as great or greater chronicity in patients suffering from pulmonary tuberculosis. The more such evidence is accumulated the better it will be for the subject of the treatment of the malady, for it will give support to those who, whilst giving fullest credence to the benefits to be derived from sanatorium treatment, feel that after hygienic rules have been learnt, the patient may in suitable cases carry out the cure of the disease in his or her ordinary surroundings and whilst carrying out the duty of a wage-earner.

\section{SOME OF THE MORE RARE CAUSES OF THE ACUTE ABDOMEN.'}

\section{BY WILLIAM HENRY BATTLE, F.R C.S. ENG.,} SURGEON TO ST. THOMAS'S HOSPITAL.

AT intervals we meet with cases presenting acute abdominal symptoms, which resemble rather closely some of those manifested by the more common examples of perforation of the hollow viscera, so that a diagnosis of one of these accidents may be wrongly arrived at. When the abdomen is - opened, however, the lesion expected is not found, and we have to search for changes which may indicate that a more unusual and perhaps unsuspected disease is present.

Aoute Hamorrhagic Pancreatitis.

In this disease, which is most difficult to diagnose, the symptoms are referred to the upper abdomen, and, as they are very urgent and accompanied with much pain, early operation is generally permitted if suggested to the patient. Yet on opening the peritoneal cavity there may be nothing abnormal presented at first sight, whilst the blood-stained fluid usually present, being small in quantity, may be overlooked as a sign of disease. And in the early stage of this illness not only may the effusion of blood be of small quantity and possibly unnoticed, but there may be no fat necrosis, the only sign which can absolutely be relied on at the time. A good illustrative case was the following.

A widow, aged 57, was sent to me at St. Thomas's Hospital by Dr. G. Brebner Scott, of Brixton, for an acute abdominal illness on Feb. 23rd, 1909. At 6 P.M. on the previous day she had complained of great pain in the abdomen. She said that it began on the right side and spread rapidly to the left, and also extended upwards to the right lower ribs. Vomiting came on at once, and she could keep nothing down subsequently. Her bowels had acted naturally the previous morning. There was no bistory of biliary colic or of injury, and she had been quite well until this illness. She was a well-nourished woman, who still complained (at 6 P.M. on Teb. 23rd) of abdominal pain. This was now general, all over the abdomen. She looked ill, had a pulse of 110, and a temperature of $101^{\circ} \mathrm{F}$. The abdomen was distended, generally hard to the touch, and very tender, but not specially so in the iliac fossa. On percussion there was patchy dulness, both in front and on the lateral aspects of the dibdomen, but not in the flanks. No abnormal swelling could be feit, but the wall of the abdomen was fat. Her tongue was dry; the bowels were not acting. Operation was decided upon, and an incision made on the right side through the rectus muscle. When the peritoneum was opened good deal of blood-stained fluid escaped. There was no lymph on the peritoneum, but the omentum appeared somewhat thick and infiltrated, whilst in two or three places there was fat necrosis. The pancreas appeared harder than usual and enlarged. The gall-bladder was normal; no stone could be felt either in it or in the biliary passages. The small intestine on the right side was distended. The peritoneum was washed out with normal saline solution and the incision was closed. The abdomen was closed because the

1 Abstract of a paper read before the Derby Medical Society on Nov, 8th, 1910. mount of fluid did not appear to increase during the operaion, and the peritoneum was quite clean. She was relieved by the operation, but on the following evening her temperature rose again to $100^{\circ}$ and pulse to 136 , so the incision was reopened, more fluid evacuated, and a drainage-tube put in. The following day Cammidge's test (o) was reported as positive. The patient was very ill for some days, and at one time appeared very flushed, weak, and despondent. Drainage was continued until March 10 th, about a fortnight, after which she gradually improved. It is not necessary here to give any further details of the case. She left hospital on April 20th, and has since had good health, having quite recovered.

This case is encouraging inasmuch as it was a successful one; unfortunately, successes are not numerous in the practice of any one surgeon, however large his experience. There have been five cases under my care within recent years, all of which were submitted to operation, but this is the only one in which a full success can be claimed. But even when operation does not save life, it relieves of the agonising pain which is so very distressing in most. The history of alcoholism was not obtained in all. As a rule, the movements of the body were more free than they are after acute perforations of intestinal ulcers. In one case the patient (a female lunatic) was found to be lying on ber side, and that position is not assumed in many acute abdominal conditions.

Fitz wrote of acute pancreatitis a few lines to which I may draw attention: "Acute pancreatitis is to be suspected when a previously healthy person or a sufferer from occasional indigestion is suddenly seized with violent pain in the epigastrium followed by vomiting and collapse, and in the course of 24 hours by a circumscribed epigastric swelling, tympanitic or resistant, with slight rise of temperature." I should say that the patient is commonly an adult of more than 40 years of age, well nourished, and even fat, apparently in good health until seized with a sudden attack of severe abdominal pain. Dr. C. R. Box has pointed out to me that the majority are females, and that this corresponds to some extent with the greater frequency of gall-stones in women.

On examination I have found that the abdomen has been more resistant generally than it should have been, but not rigid. There has been a diffused superficial tenderness, especially on unexpected light palpation; the general resonance over the abdomen has been rather patchy in character without evidence of free fluid, whilst the movements during respiration have been good. In all the pulse has been rapid, there has been anxiety, and not infrequently a flushed face, whilst there has been a rise of temperature. As Dr. A. Elliot reminds us in his interesting paper on this subject ${ }^{2}$ (to which I am indebted), the cause of the rapidly fatal ending in acute hæmorrhagic pancreatitis is not always easy to determine. It has been put down to hæmorrhage, but many cases cannot be so explained, for in a considerable number the amount of extravasated blood has been comparatively small. Nor is it probable that the sudden disturbance of the whole or part of the pancreas is a sufficient explanation. Some have ascribed the fatal result to shock.

The other acute abdominal conditions which most clearly resemble it clinically are: (1) perforations of the stomach or daodenum; (2) perforations of - the appendix; (3) acute intestinal obstruction; (4) acute cholecystitis with perforation of the gall-bladder; (5) acute irritant poisoning; and (6) very rare conditions, such as embolism of the mesenteric artery, rupture of a liver abscess or hydatid, \&c. Of these it is most commonly put down to perforation of the stomach, but in the anterior perforation, although we have the sudden onset of intense pain, there is rigidity of the rectus muscles in the epigastric region, loss of liver dulness, with the rapid effusion of free fluid down the right side of the abdomen. When a posterior perforation of the stomach has occurred the symptoms are mostly referred to the lesser sac, so that they may be more difficult to differentiate. In gastric perforations the vomiting may, but does not usually, continue, and may indeed be absent, whilst there is a history of gastric symptoms pointing to chronic disease of the stomach or duodenum.

In aoute appendicitis, although the pain is referred to the umbilicus at the beginning of the attack, within a short time there is tenderness in the iliac fossa and the pain moves to that part. The muscles hecome rigid over the iliac fossa,

2 Transactions of the West London Medico-Chirurgical Society, Oetober, 1910. 
and localised dulness on percussion may be demonstrated, whilst if the appendix is placed unusually high on the right side, although the resemblance to acute hæmorrhagic pancreatitis is more close, there is still the differentiating rigidity and localised dulness.

In aoute intestinal obstruotion high up, possibly from a band, the pain is paroxysmal, vomiting very urgent, and the initial shock marked. Fæcal vomiting occurs early and obstruction is complete, no flatus being passed. Abdominal distension is but slight if any is present.

Gall-stone oolio and chuleoystitis are not infrequently diagnosed, more especially as there is so often the history of gall-stone colic in the past life. In the more acute cases of perforation of the gall-bladder a tender swelling may have been noticed in the gall-bladder region for some timeperhaps for days-before the onset of urgent symptoms.

Cases of poisoning rarely lead to any real difficulty. In acute metallic poisoning the purging is a prominent feature, and the discovery of the poison in the vomit places the diagnosis beyond doubt. In ordinary food poisoning there is usually more than one patient affected ; diarrhoea is present as well as the vomiting, and cramp-like pains are a frequent accompaniment.

In acute hæmorrhagic pancreatitis if the abdomen is opened within 24 hours the amount of blood-stained fluid will be small, and may be supposed to have run from the external nound into the peritoneum; again, at this stage, it may be difficult to find any of the yellow points of fat necrosis. In any case in the adult where nothing is found in the more asual places to account for acute abdominal symptoms, search should be made for these areas, which are of small size, somewhat irregular outline, and yellowish-white in colour. I have been struck with the sodden state of the great omentum in some early cases, even when there have been very few points of fat necrosis visible. It has been of a greyish appearance, and has lost elasticity. There may be no evident swelling of the pancreas. If nothing is found which explains the sudden acute attack, it will be best to put in a drainage-tube for a few hours - for I can but think that some of the cases of abdominal section which have been closed "because nothing was found," have been of this nature, and without free drainage for a time the result of interference in most of them is worse than useless. A discharge which at first is of a reddish colour and varying amount will generally come away through the tube; this becomes of a yellow tint, and possesses a peculiar mawkish smell, and must not be permitted to accumulate within the peritoneal cavity, however small in quantity it may be. These patients are rarely good subjects for abdominal section, and I believe better results will be got on the average from simple drainage than from a more elaborate operation, such as incision of the pancreas, \&c., possibly with drainage of the gall-bladder. Most of them will not stand the additional manipulations required, with the prolongation of the period of anæsthesia; it is possible, however, to do more if the patient is seen early in the attack, is in fair condition, and not too fat. The ideal operation is to incise the pancreas, with due regard to the duct and main vessels, and establish a direct route for the drainage preventing the escape of fluid into the surrounding peritoneal cavity, as in cases of rupture of the organ. Unfortunately the action of the secretion from the gland, if much escapes, is very destructive on the tissues with which it comes in contact, and if the flow is profuse you will find it difficult, or even impossible, to prevent actual digestion of parts.

\section{Acute Dilatation of the Stomach.}

This is a rare condition, the origin of which is sometimes doubtful; it may follow an operation involving the peritoneum. An attack starts with copious fluid vomiting, epigastric pain and distension, which becomes diffused the action of the bowels is irregular; signs of extreme collapse are present. Towards the end of a severe case complete atony of the stomach may lead to cessation of the vomiting. Of physical signs, the most valuable, when it is present, is succussion, but it is important to remember the possibility of the occurrence of such a condition in the acute abdomen. Unless relieved by evacuation of the stomach contents it usually proves rapidly fatal. The extreme distension of the abdomen and generally severe condition may lead to a diagnosis of acute peritonitis, or if there is constipation intestinal obstruction may be thought to be present. I will give two contrasting examples, one occurring in the course of an acute pulmonary attack, the other secondary to an abdominal operation and associated with general intestinal distension.

(I.) On Jan. 21st, 1903, I was asked to see, with Dr. Michael J. Bulger, a patient aged 15 years. The history of the case. was as follows. Dr. Bulger was called to see her on the 19th, when she complained of pain under the left breast on breathing, which was increased by taking a deep breath; there was slight expectoration tinged with blood. Over the painful area there was some dulness, increased vocal resonance, and crepitation on respiration. On the 20th the patient was much easier. Temperature, $102^{\circ} \mathrm{F}$.; pulse, 96 ; could take food easily, bowels acting. She was the subject of angular curvature of the dorsal spine, the result of old tuberculous disease. On the 21st she began to vomit at about 7 A.M., the vomited material being of a bilious character and yellow in colour. The bowels acted at $8 \mathrm{~A} . \mathrm{M}$. The pain in the side was much better, but the constant vomiting masked all other symptoms. Temperature, $99^{\circ}$; pulse, 80 . Nothing relieved the sickness. The abdomen was retracted, dull all over, and without tenderness on pressure. At 6 P.M. she was rather collapsed, the vomiting continued, and now she was bringing up a black, tenacious fluid. She had complained of no pain since the vomiting came on, but the abdomen was becoming disterded. About 11.30 P.M., when I saw her with Dr. Bulger, the abdomen was somewhat distended, but not markedly so, dull on percussion all over the front and down the left flank to Poupart's ligament. No dulness was present in the right flank. A well-marked thrill of fluid could be felt in the lower part and to the left. There was no rigidity. The pulse was rapid, face pale and sunken, tongue black and dry, whilst there was frequent vomiting of a black, tarry fluid. $\Delta \mathrm{n}$ incision in the middle line showed a greatly distended stomach, the lower margin of which passed down to the pubes; it was bluish in appearance and flattened. All the intestines were empty. There was no free fluid. Distension apparently ceased at the third part of the duodenum, and no pressure could empty the contents of the stomach along this part. A tube was put in, and the opening sutured to the abdominal wall. Much fluid was drained off from the stomach by this tube, and romiting ceased; but very little relief was afforded, and the patient died on the following day, apparently from exhaustion.

(II.) This is the other example. A woman, aged 27 years, came under my care at St. Thomas's Hospital, sent to me by the late Dr. Heath of St. Leonards-on-Sea, on Nov. 7th, 1901, for a swelling in the abdomen, which had been noticed nine jears, and was said to be increasing. On the 12th cœliotomy was performed, the diagnosis of ovarian cyst confirmed, and the tumour removed in the usual manner. On the first and second days after the operation the patient's pulse was about 110, and the temperature rose from $101^{\circ}$ to $103^{\circ} \mathrm{F}$. The abdomen became increasingly distended; there was no vomiting beyond that directly following the anæsthetic. A week after the operation there was evidence of slight suppuration in the abdominal wound, and pus was evacuated with a director. There continued to be great distension of the abdomen and much discomfort. On the 21st Dr. C. R. Box saw the case with me. The epigastric area was then very prominent and a ringing coin sound could be obtained over this area and extending downwards to the iliac crests; marked succussion was elicited on shaking the patient; there was no vomiting. Lavage of the stomach was commenced and carried out twice daily from this time. 26 days from the operation parotitis developed, associated with a septicæmic temperature and severe diarrhœa, and for some time this was uncontrollable. Antistreptococcic serum was given; a marked rash followed two days after its administration, but it was without apparent effect on the disease. The distension of the abdomen did not appreciably diminish, and with a high temperature and the diarrhoea it continued for about three months; much cedema of both legs and the lower part of the abdominal wall supervened. Some peristalsis in the region of the umbilicus was occasionally seen, and the stomach still showed the physical signs of dilatation. On August 12th, 1902, the patient left the hospital. The gastro-intestinal functions had become normal, but the odema in the lower part of the body, due (presumably) to thrombosis of the inferior vena cava, was still present. Seen again in January, 1903, her general health was good, though evidence of thrombosis persisted, there being some cerlema of the ankles, with dilatation of the veins over the lower part of the abdomen. 
The subsequent history of the second case is very interesting. The patient was readmitted to St. Thomas's Hospital under my care on Nov. 19th, 1907, for another abdominal swelling. It was stated that her general health had been good until a fortnight before, but that during that time she had suffered from pain in the stomach and swelling, but no vomiting. The abdomen was a good deal distended and tense on admission, the superficial veins dilated, chiefly in the lower part, and there were numerous lineæ albicantes in the same region. A dull rounded area was present reaching almost to the umbilicus from the pelvis. This was fluctuating and tender, whilst around it the intestines were distended and tympanitic. Her temperature was slightly raised. She was kept in bea for some time in order to give the inflammatory state a chance of quieting down, but the distension did not appreciably diminish. On Dec. 4th an incision to the left of the mid-line was made, and an inflamed ovarian cyst was removed. The pedicle was long and had been twisted three times from left to right, this rotation probably resulting from the movements of the ovar-distended pelvic colon. The cyst was very adherent to the omentum, but not suppurating. It was an ordinary multilocular cyst. The gut was very much distended, the sigmoid being about 5 inches in diameter when examined in the wound. The intestine was not punctured, as the condition was regarded as temporary in character. Much flatulent distension of the abdomen continued, not involving the stomach; many remedies were tried, but until the employment of the interrupted current late in December no definite effect appeared to have been produced by them, but the distension suddenly subsided on the 25 th of that month. There was no suppuration or rise of temperature after the operation. The unusual amount of distension of the intestines present at the time of the second admission and the dificulty in getting rid of it after operation are especially interesting in a patient with this history. On this occasion there was no suppuration either before or after the operation, get the distension was extreme, and suggested that the nervous element was an important factor in its causation. The rapid recovery on the use of the interrupted current confirms this view. We know how marked the "reflex" effect may be sometimes of an injury to the abdomen unattended with obvious lesion, also the great distension of the abdomen which may ensue on the mere application of a ligature to the neck of a hernial sac in the operation for radical cure. In a patient once under my care a condition of rapid distension of the abdomen with pain, vomiting, and a temperature of $103.6^{\circ} \mathrm{F}$. ensued, with a collapse which excited alarm. Appropriate remedies soon produced a change for the better and the case ran the usual aseptic course without further symptoms.

The cases narrated above are both of them examples of acute dilatation of the stomach but present many points of contrast. In the first case the stomach had become a mere fluid-cuntaining sac with a thin wall, which at the time of the operation was lying over the front of the intestines and gave a dull note on percussion across the middle line, an area which is resonant in all other conditions of the acute abdomen. There was most certainly no gaseous accumulation, and until quite the last stage there was no distension of the abdomen. It is difficult to account for the distension unless we accept that it was a paralysis due to some toxic condition associated with the patch of inflammation of the left lung found by Dr. Bulger when he first saw the patient. Spinal deformity has been noticed in other cases of acute dilatation, but when not associated with the application of a plaster jacket it is difficult to understand how it could have much influence on the production of such an acute and fatal affection.

Dr. W. B. Laffer collected a series of 217 reported cases, and of these 382 per cent followed operations, usually one on the abdomen. The earlier notes of Case 2 were published by Dr. Box and myself in THE LANCET on account of its rarity, and as an encouragement in the treatment of such desperate conditions. We are inclined to put its occur rence down to some toxic absorption from the wound although the amount of suppuration was neither acute nor extensive. It is probable that she owed her recovery to the fact that the distension was general and not absolutely confined to the stomach ard duodenum. Dr. Laff $\mathbf{x}$, from an analysis of his series of cases, writes: "The pathology and modus operandi of acute dilatation of the stomach and gastrnmesenteric ileus is not definitely known, bit the experimental, clinical, and pathological evidence points to a primary innervation disturbance affecting the gastric nerves or their centres in the brain or cord. It has not been proved that the compression of the duodenum by the root of the mesentery is the primary cause of the so-called arteriomesenteric ileus." Thus agreeing with Dr. Box and Mr. C. S. Wallace in their opinion. ${ }^{3}$

\section{Some Neuroses which may Cause Symptoms of Urgenoy.}

Hamorrhage from the stomach. - No surgeon has any doubt that operative treatment is sometimes absolutely necessary in hæmorrhage from gastric or duodenal ulcers. It may be the only means of saving life, but the indications for its performance should be clear and definite. In some cases it may be possible to find and deal with the exact cause of the hæmorrhage ; in others it will only be possible to treat the distension of the stomach (by gastro-enterostomy) on which the occurrence of the bleeding so frequently depends. It may be the wiser plan, when possible, to perform the operation of gastro-enterostomy, although the local trouble has also been directly treated.

In dealing with these cases it may be advisable to remember the possibility of the bæmatemesis being of hysterical origin for such a condition is always amenable to medical treatment, and in my opinion should not be submitted to operation under any circumstances. The history of the case here given not only supports this statement but shows in a marked degree the ills that may follow ill-advised interference.

A woman, aged 29 years, was sent to me by Mr. Frank Boxall of Rudgwick in September, 1902, for varicose veins of the left leg which were causing her pain when standing. She was admitted to St. Thomas's Hospital (Mr. T. Guthrie was house surgeon), and 'Trendelenburg's operation with excision of some of the more prominent veins in the calf was performed. In her past history it was stated that she had been in another hospital a short time before for symptoms which were regarded as indicating the presence of a gastric ulcer. One night she developed acute symptoms, which were supposed to have been due to perforation of the ulcer, and an exploratory incision was made in the epigastric region by a surgeon, who found nothing but a normal state of the stomach; there had been no perforation. From the history this attack was supposed to have been hysterical. During her stay in hospital this opinion was confirmed by the fact that in the earlier days after her admission, when she was looking somewhat anxious in the face, she again gave an exhibition of perforation. She complained of acute pain in the epigastrinm, the upper abdomen became suddenly distended, and the muscles appeared tense. There was, however, no change in her appearance, the pulse-rate, or temperature, and other symptoms were not in agreement with perforation; we had also the history to go upon, and the condition quickly improved when no special treatment was given. This patient left St. Thomas's about a fortnight after the operation for the veins, but returned in 1904 on account of hæmatemesis. She was vomiting daily, without any relationship to food, large quantities of fluid, in which there was a good deal of blood of dark colour, evenly diffused. In spite of the fact that this continued for a month without cessation, she showed no signs of anæmia, and always presented a smiling face to the world. No particular drug was given to arrest the bleeding, which was regarded as of hysterical origin. When the hæmatemesis had ceased for a few days and she had become brigbt and cheerful she was sent home In about tbree months' time she was sent back to the hospital with another attack of hæmatemesis of similar character, from which she recovered in from three to four weeks, and returned to her home quite well. It was some months before anything further was heard of her, and then it appeared that she had again developed hæmatemesis when the influence of the hospital had passed off, and this time her friends sent her to a hospital "where there was a surgeon who would operate." Her next admission to St. Thomas's was on July 19th, 1905, when she was found to have a fæcal fistula, which communicated with the transverse colon and was situated at the lower part of a scar, through which, it was stated, her 
stomach had been operated on. We were informed by letter that although no ulcer or cause for the hæmorrhage was found at the examination, it was "thought" by the surgeon that there was an ulcer in the duodenum. She said that after the operation she did very well until the tenth day, when it was found that the milk which she was taking came through into her dressings. A second operation was done and the milk no longer came through the wound, but in ten days' time fæcal matter appeared when she took medicine, and freal fluid came through if she had an enema administered. The abdomen was opened in the middle line below the old scar and a lateral anastomosis of the large bowel above and below the fistula done. There were many adhesions. Recovery from this operation was quite aneventful, the fistula was allowed to close and, when she left the hospital, it was of about the size of a wooden match. She left at her own request. Readmission was sought in January, 1906, because she said that the escape of gas from the fistula was troublesome and caused offence to patients when she was nursing them. There was now a fistula of about the size of a cedar pencil, and as the bowels were acting well there appeared no reason why this should not be permitted to close. Accordingly a dressing was placed over it, and secured in position by means of broad strips of rubber strapping. The fistula closed to some extent, but we could not feel sure that it was not kept open in some way by mechanical means at the command of the patient. A smaller dressing was then applied, and this was covered and held in position by means of collodion. After this was applied she complained of excruciating pain and said that she could not possibly bear the agony of it. It was not, however, removed for a week, when the fistula had completely closed. I may perhaps mention that the fistula was found to have become distinctly larger after she had had a bath without the presence of a nurse; this was before the collodion was applied.

We were for a time under the impression that the case was now completed, but in March, 1909, the patient again came into the hospital during the cleaning of a charitable institution to which she had gained admission. A fæcal fistula had formed at the site of the former one, and she refused to have anything done with a view to closing it. When questioned as to the formation of this fistula she said that an abscess had come and bad burst, leaving the fistula behind it, but there is a strong possibility that it did not form in this manner. If it had been closed, and this would soon have occurred under simple treatment, for there was a free normal passage for the fæces, she would no longer have been eligible for the institution in which she had now been received. I may add that her expression was that of a neurotic, and the diagnosis of hysteria was confirmed in many ways.

It was surely unnecessary to perform a gastrotomy for the relief of hæmatemesis in a case with this history. Gardini ${ }^{4}$ has given the account of a case of similar origin. A girl, aged 22 years, had suffered from gastric symptoms for six years, and almost daily volniting of blood for five months or more; in that instance the mucous membrane of the stomach is said to have been tinged, hypertrophic, and of a red colour, but there was no evident cause for the hæmorrhage. The patient was apparently cured by the operation. Gastric hromorrhage has sometimes a purely nervous origin; sometimes it is simply a form of vicarious menstruation and has a relationship to the menstrual periods, as well as to emotional and constitutional disturbances and injury. ${ }^{5}$

Those of us who are engaged in doing much operative work must recognise what is almost a disease amongst a certain class of patients, chiefly those with abdominal symptoms-the desire for operations. It is not very unusual to see many scars on the abdomen and yet a continuance of symptoms.

Enteraspasm.-By this term is now recognised a condition in wnich there is a spastic contraction of the muscular wall of some part of the intestines; there is no obvious structural change in the bowel, and the phenomena are usually regarded as being dependent upon some abnormal action of the nervous mechanism. The spasm may give rise to symptoms of varying intensity, from those of chronic constipation to such as simulate acute intestinal

${ }^{4}$ Clinica Moderna, May, 1905; Brit. Med. Jour. Epitome, August, 1905 . obstruction. Dr. H. P. Hawkins drew attention to the condition in 1906," and I will quote from some of the conclusions he then set down. "Symptoms usually manifest themselves in patients during the active period of life ; they appear with about equal frequency in the two sexes. The individuals affected are usually of a neurotic type and often of sedentary habits." Opportunity for direct observation of the spasm of the bowel does not often occur, but Dr. Hawkins thinks that the colon is more often affected than the small intestine. The pain in the subacute cases is sometimes localised in the right iliac region, and so appendicitis may be simulated.

I need here only consider the severe cases giving rise to symptoms which suggest the necessity of immediate operative interference. Sometimes the resemblance of the condition to intestinal obstruction of organic origin, or even to general peritonitis, may be so close that the mind of the observer is left in doubt as to the right diagnosis, and exploration of the abdomen will be the only sound course to pursue. Points which will be helpful in arriving at a decision are the presence of the trouble in highly strung, nervous individuals, and a history of previous attacks of abdominal pain similar in character and which have passed off without operation.

In a recent case operated on for me by the resident assistant surgeon, Mr. L. E. C. Norbury, the patient was a woman aged 40 years, for whom I bad removed gall-stones about two years previously. Her symptoms were those of acute intestinal obstruction, and the spasmodic contraction affected much of the small intestine. She is a typical neurotic in appearance. I have met with the condition as a mere localised affection of the splenic flexure in more than one instance; here the patients have been overworked and anxious men of over 45 years of age.

Harley-street, W.

\section{A CASE OF}

INTERSTITIAL GESTATION AT THE THIRD OR FOURTH MONTH WHERE THE SAC WAS REMOVED UNRUPTURED WITH THE BODY OF THE
UTERUS BY ABDOMINAL
HYSTERECTOMY.

\section{BY ARTHUR H. N. LEWERS, M.D. LOND.,} F.R C P. LOND.,

SENIOR OBSTETRIC PHYSICIAN TO THE LONDON HOSPITAL.

THE patient, a married woman, 29 years of age, was admitted into the London Hospital on July 17th, 1910. She had been married for three years, and had had one child 11 months previously and one miscarriage three months after marriage. She had suckled the baby up to a fortnight before her admission. Soon after the confinement there was an interval of amenorrhœa lasting six weeks, but after that the periods came on and continued to occur regularly (although she was suckling) up to the middle of April, 1910. Since that date she had "seen nothing." Ten days before admission to hospital she had noticed a lump in the lower abdomen on the left side. Three days before admission she was seized during the night with sudden attacks of severe pain in the region of the lump. When she was brought up to the hospital she was evidently acutely ill, but as the cause of her illness was uncertain till a careful history had been obtained she was admitted in the first instance on the surgical side. When the circumstances were more fully investigated the case was thought to be probably obstetric, and it was therefore transferred to the obstetric wards in the early morning of July 18th, where I saw her at about $2 \mathrm{~A}$.M. The pulse was then 120 and the temperature $97.5^{\circ} \mathrm{F}$. She was suffering from very acute pain in the lower abdomen, but she was not anæmic, nor was there any vaginal bleeding. The mass in the left iliac region was felt, but could not be very thoroughly examined as there was great tenderness, and the muscles were rigid. On vaginal examination the cervix was soft, and the mass to the left appeared to be closely connected with the uterus (which was enlarged) and perhaps part of it. The history of 